\title{
Hemangioma of the Prostate: A Case Report and Literature Review
}

\author{
Hsu Fu-Kai ${ }^{1}$, Hung Siu-Wan ${ }^{2}$, Wu Chih-Ying ${ }^{3}$, Chiang Heng-Chieh ${ }^{4}$ and Chiu Kun-Yuan ${ }^{5 *}$ \\ ${ }^{1}$ Taichung Veterans General Hospital, Taichung, Taiwan \\ ${ }^{2}$ Department of Radiology, Taichung Veterans General Hospital, Taichung, Taiwan \\ ${ }^{3}$ Department of Pathology and Laboratory Medicine, Taichung Veterans General Hospital, Taichung, Taiwan \\ ${ }^{4}$ Division of Urology, Department of Surgery, Changhua Christian Hospital, Changhua, Taiwan \\ ${ }^{5}$ Division of Urology, Department of Surgery, Taichung Veterans General Hospital, Taichung, Taiwan
}

"Corresponding Author: Chiu Kun-Yuan, Division of Urology, Department of Surgery, Taichung Veterans General Hospital, Taichung, Taiwan, Tel: +886-4-23741215; Email: chiu37782002@yahoo.com

Received: 01 September 2017; Accepted: 18 September 2017; Published: 22 September 2017

\begin{abstract}
Hemangioma of the prostate is extremely rare. Here we described a 69-year-old man who had lower urinary tract symptoms (LUTS) for 5 years, treated as benign prostate hyperplasia (BPH) by Diode laser enucleation. Massive bleeding developed during the procedure with subsequently recurrent urine retention due to blood clot accumulation off and on for the following 3 years. Selective arterial embolization and repeated bladder irrigation failed to control the continuous bleeding, which finally caused obstructive uropathy. Cystoprostatectomy was then performed and the pathological result proved cavernous hemangioma of the prostate.
\end{abstract}

Keywords: Hemangioma; Laser therapy; Lower urinary tract symptoms; Prostate

Abbreviations: BPH: Benign Prostate Hyperplasia; CT: Computed Tomography; LUTS: Lower Urinary Tract Symptoms; PSA: Prostate Specific Antigen; TURP: Transurethral Resection of the Prostate

\section{Introduction}

LUTS is the symptoms related to storage, voiding, or post micturition. It is primarily attributed to BPH in elderly men [1]. Hemangioma, a vascular malformation, is an extremely rare etiology of LUTS. When hemangioma was 
large enough, it can lead to LUTS. Gross hematuria is the cardinal symptom of urinary tract hemangioma, but it is difficult to confirm the diagnosis by conventional radiographic examination [2]. If prostatic hemangioma presents with LUTS rather than hematuria, we may easily confuse it with BPH. Transurethral resection of the prostate (TURP) had been used to treat the prostatic hemangioma with transient complication of a slow stream and initial voiding difficulty [3]. Selective arterial embolization had been applied on prostatic hemangioma treatment with only one episode of hematuria after the procedure [4]. The perineal intracapsular prostatectomy was performed in a reported case, but the patient was dead due to uncontrollable bleeding during the operation [5]. Thus, misdiagnosis of prostatic hemangioma can lead to a tragedy. We present a case of prostatic hemangioma treated with Diode laser enucleation complicated with repeated bleeding and obstructive uropathy for 3 years.

\section{Case Report}

A 69-year-old man had LUTS since 5 years ago. The rectal examination showed a huge prostate with multiple nodularity. Serum prostate specific antigen (PSA) level was $8.74 \mathrm{ng} / \mathrm{ml}$. Transrectal ultrasound disclosed the prostate was $89 \times 71 \times 76 \mathrm{~mm}$ with an internal $45 \times 24 \mathrm{~mm}$ cystic lesion (Figure 1). Abdominal computed tomography (CT) revealed an enlarged prostate with a well-enhanced glandular hyperplasia structure and peripheral enhancement similar to "ring of fire" in the delay phase (Figure 2). Although prostate cancer cannot be ruled out, the patient decided to receive Diode laser enucleation of the prostate. Massive intraoperative bleeding was noted and Foley's catheter traction was performed. After the procedure, LUTS was improved but intermittent hematuria with recurrent urine retention bothered him in the following 3 years. Bladder irrigation by cystoscopy and even cystoscopic evacuation of hematoma were performed for several times. This patient also received selective arterial embolization for twice but in vain. He had obstructive uropathy with acute renal failure (serum creatinine level 12.0 $\mathrm{mg} / \mathrm{dl}$ ) developed 4 years later. Bilateral percutaneous nephrostomy were introduced and the renal function recovered (serum creatinine level $2.5 \mathrm{mg} / \mathrm{dl}$ ). The serum PSA increased to $12.49 \mathrm{ng} / \mathrm{ml}$ and non-contrast CT revealed marked enlargement of the prostate gland due to hemorrhage. Laparoscopic radical cystoprostatectomy with ileal conduit reconstruction was performed. The pathology proved cavernous hemangioma with hematoma of the prostate (Figure 3) and incidental adenocarcinoma of the prostate (Gleason's score: $3+3=6$ ).

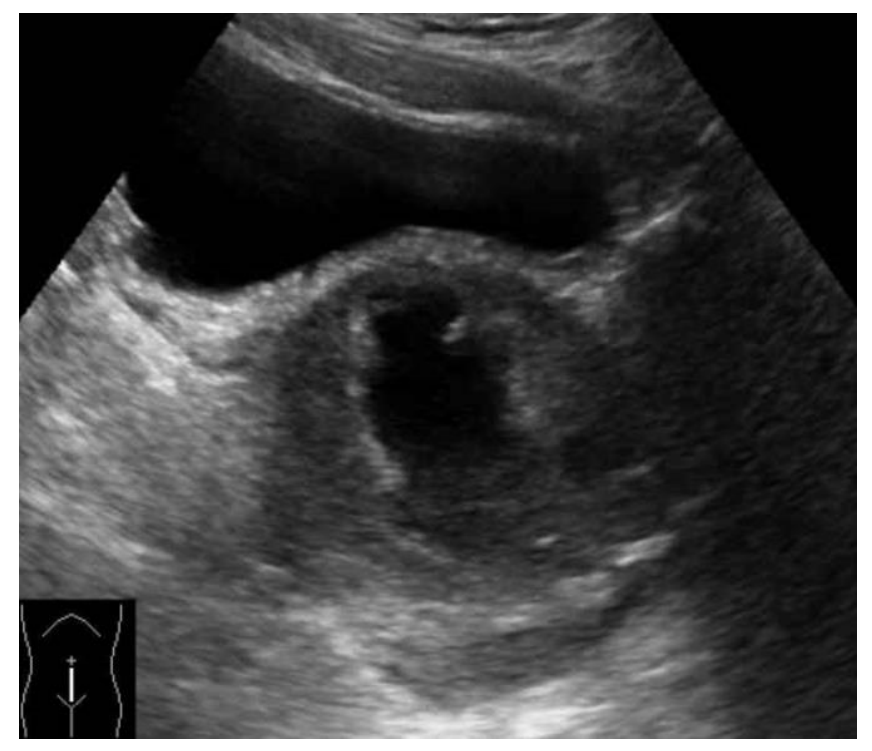

Figure 1: Ultrasonography demonstrated a huge prostate with a $45 \mathrm{~mm}$ x $24 \mathrm{~mm}$ multiloculated cystic 


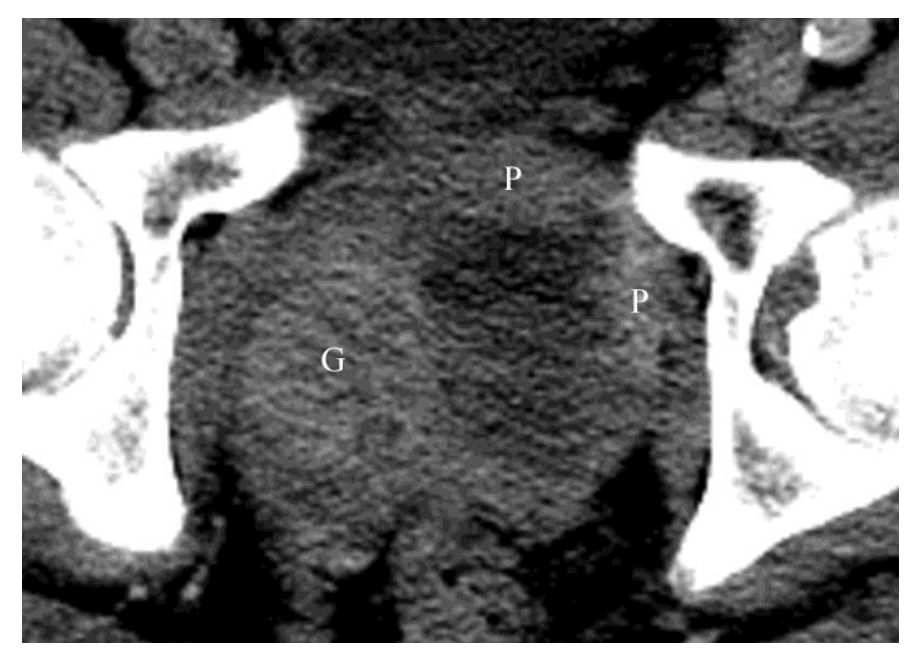

Figure 2: Delay-contrasted CT revealed a hypoattenuated lesion in left lobe with peripheral enhancement $(\mathrm{P})$ similar to "ring of fire". Note that the well-enhanced glandular hyperplasia structure $(\mathrm{G})$ on the right lobe.

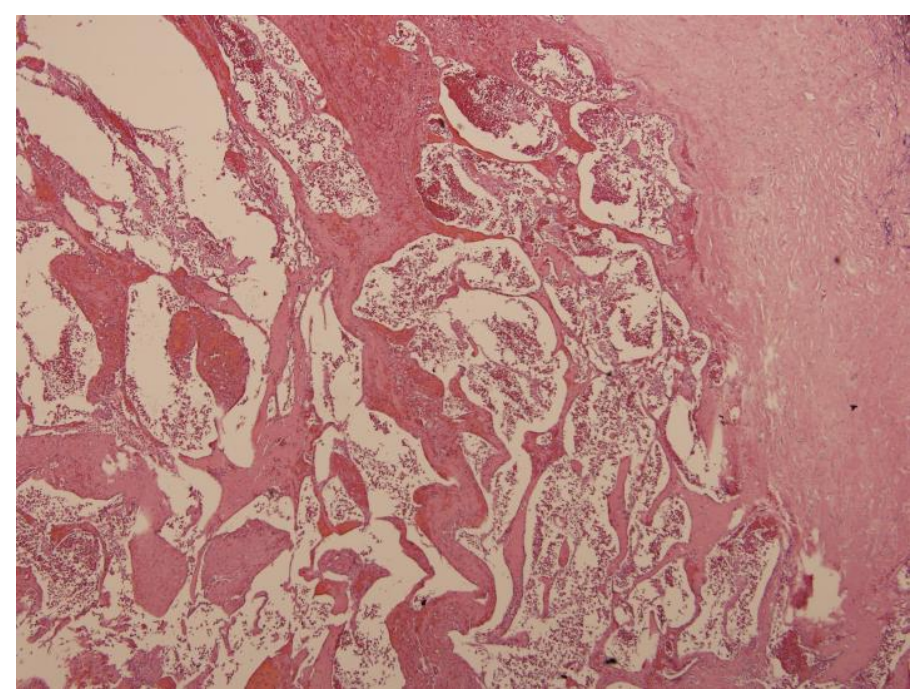

Figure 3: Irregular dilated and anastomosing vascular channels accompanied with fibrosis in prostate (H\&E, 100X) were noted under microscopic evaluation. Cavernous hemangioma with hematoma in prostate is impressed.

\section{Discussion}

Hemangioma of the urinary tract is very uncommon. Renal hemangioma is reported most frequently, then the urinary bladder. Hemangioma exclusively located in the prostate is the rarest. Hematuria is the usual symptom of hemangioma of the urinary tract [2]. Four published cases of prostatic hemangioma were reported in the literature (Table 1). The major clinical symptoms were not hematuria but LUTS. The conventional radiographic tool was not helpful for diagnosis of hemangioma. The cystoscopy showed bluish-red vascular area, compatible with hemangioma [4]. In two patients received prostectomy, one had no complication and the other was dead after operation [5, 6]. The other two patients treated with TURP and selective arterial embolism had no severe complication after procedure [3,4]. Fiorelli et al. reported a case of hemangioma located in both prostate and bladder and this patient had painless gross hematuria and hematospermia for nine years. He was treated by arterial embolization with polyvinyl alcohol [4]. 


\begin{tabular}{|c|c|c|c|c|c|c|}
\hline Case & Authors & Age & Symptom & $\begin{array}{l}\text { Pre-operative } \\
\text { image finding }\end{array}$ & Treatment & Complication \\
\hline 1 & $\begin{array}{l}\text { Rivoir et al } \\
(1953)(5)\end{array}$ & 65 & LUTS & $\begin{array}{l}\text { Cystoscopy: bladder } \\
\text { trabeculation and } \\
\text { injected mucosa }\end{array}$ & Prostatectomy & Death \\
\hline 2 & $\begin{array}{l}\text { Fiorelli et al } \\
\text { (1991) (4) }\end{array}$ & 30 & $\begin{array}{l}\text { Hematuria and } \\
\text { hematospermia }\end{array}$ & $\begin{array}{l}\text { Cystoscopy: bluish- } \\
\text { red vascular area }\end{array}$ & $\begin{array}{l}\text { Selective arterial } \\
\text { embolism }\end{array}$ & Hematuria once \\
\hline 3 & $\begin{array}{l}\text { Ristau et al } \\
(2011)(6)\end{array}$ & 69 & Asymptom & $\begin{array}{ll}\text { No } & \text { remarkable } \\
\text { finding } & \end{array}$ & Prostatectomy & No \\
\hline 4 & $\begin{array}{l}\text { Serizawa et } \\
\text { al (2011) (3) }\end{array}$ & 84 & LUTS & $\begin{array}{ll}\text { No } & \text { remarkable } \\
\text { finding } & \end{array}$ & TURP & $\begin{array}{l}\text { Slow stream and initial } \\
\text { micturation difficulty } \\
\text { for } 6 \text { months }\end{array}$ \\
\hline
\end{tabular}

Table 1: Reviewed hemangioma of the prostate.

Due to the rarity, hemangioma is usually at the bottom of the differential diagnosis list of hematuria. The hemangioma is suspected only after infection, malignancy and urolithiasis are ruled out [2]. The diagnosis of hemangioma is hardly ever established before operation. The unexplained hematuria or hematospermia is the only clue to distinguish hemangioma from BPH in the setting of hemangioma presented with LUTS. There is no consensus regarding the sonographic features of prostatic hemangioma [3]. In our case, the transrectal ultrasound revealed an intra-prostate cystic lesion with hyperechoic margin is not a typical feature shown in hemangioma of liver [7]. Once sonography shows a hypoechoic lesion inside the prostate, the color Doppler technique should be performed. It may disclose internal flow as a common sign found in hemangioma of liver [3]. Besides, contrasted CT revealed peripheral enhancement as a typical feature of hemangioma in delayed phase [7]. Hence, contrasted CT can be a useful tool for diagnosis of prostatic hemagnioma. If cystoscopy shows a bluish-red area in the bladder or prostatic urethra, prostatic hemangioma should be suspected [4]. The arteriography of internal iliac arteries is usually helpful [8]. Bryniak et al. utilized technetium-99-tagged albumin scan for diagnosis of bladder hemangioma [9].

Treatment of prostatic hemangioma includes surgical resection, laser, selective arterial embolization and radiation. Because the hemangioma can regress spontaneously, conservative treatment is another treatment of choice $[2,4,8$, 10]. In our case, the uncontrollable bleeding developed after Diode laser enucleation of the prostate, even though laser vaporization might decrease the risk of bleeding compared to traditional TURP [1]. The subsequent transurethral treatment of hematoma and selective artery embolization for prostate cannot resolve the bleeding. If the diagnosis of prostatic hemangioma was established before operation, the decision to perform TURP is not indicated due to the possibility of severe bleeding. In case of patient with huge prostate gland and cystic mass inside, with or without hematuria, the diagnosis of prostatic hemangioma should be kept in mind. The surgical intervention should be carefully discussed to prevent the massive bleeding, even though the incidence of prostatic hemangioma is rather low. 


\section{Conflicts of Interest}

The researcher claims no conflicts of interests.

\section{References}

1. Oelke M, Bachmann A, Descazeaud A, et al. EAU guidelines on the treatment and follow-up of nonneurogenic male lower urinary tract symptoms including benign prostatic obstruction. European urology 64 (2013): 118-140.

2. Jahn H, Nissen HM. Haemangioma of the urinary tract: review of the literature. British journal of urology 68 (1991): 113-117.

3. Serizawa RR, Norgaard N, Horn T, et al. Hemangioma of the prostate--an unusual cause of lower urinary tract symptoms: case report. BMC urology 11 (2011): 4.

4. Fiorelli RL, Koolpe HA, Klaus RL. Use of polyvinyl alcohol in treatment of bladder and prostatic hemangioma. Urology 38 (1991): 480-482.

5. Rivoir J, Kaslaris E. Beitrag zum prostatahämangiom. Der Chirurg; Zeitschrift fur alle Gebiete der operativen Medizen 24 (1953): 426.

6. Ristau BT, Tomaszewski JJ, Parwani AV, et al. Incidentally discovered capillary hemangioma of the prostate. The Canadian journal of urology 18 (2011): 5914-5915.

7. Vilgrain V, Boulos L, Vullierme MP, et al. Imaging of atypical hemangiomas of the liver with pathologic correlation. Radiographics : a review publication of the Radiological Society of North America, Inc. 20 (2000): 379-397.

8. Shabaik A. Nonepithelial tumors and tumor-like lesions of the prostate gland. Critical reviews in clinical laboratory sciences 40 (2003): 429-472.

9. Bryniak SR, Morales A, Challis T. Assessment of cavernous hemangioma of bladder by technetium-99tagged albumin scan. Urology 13 (1979): 289-291.

10. de Leon JP, Arce J, Gausa L, et al. Hemangioma of the prostatic urethra: holmium laser treatment. Urologia internationalis 80 (2008): 108-110. 